VOLUMEN 37 | No 2 | SEPTIEMBRE 2019

FECHA DE RECEPCIÓN: : 03/07/2019 FECHA DE APROBACIÓN: 17/07/2019 FECHA PUBLICACIÓN: 10/09/2019

1. Universidad de Cuenca

2. Libre ejercicio

\begin{tabular}{c|l} 
Artículo & $\begin{array}{l}\text { Original } \\
\text { original }\end{array}$ \\
Article
\end{tabular}

DOI: https://doi.org/10.18537/RFCM.37.02.05

Correspondencia:

jose.ortizQucuenca.edu.ec

Dirección:

Calle Vega Muñoz \# 19-35

Código Postal:

010208

Teléfonos:

07-2841322 / 0993767208

Cuenca - Ecuador

\section{Parámetros dietéticos y alimentación habitual de adultos mayores. Cuenca 2018 $-2019$.}

\author{
Dietary parameters and habitual diet of older adults. Cuenca \\ $2018-2019$.
}

Ortiz Segarra José Ignacio', Freire Argudo Ulises Vicente1, Tenemaza Alvarracín David Fernando1', Cobos Torres Ángel Ignacio', Ortiz Mejía Pedro Fernando ${ }^{2}$

\section{RESUMEN}

Objetivo: conocer los parámetros dietéticos y hábitos alimenticios de adultos mayores atendidos en centros de salud de primer nivel, en la ciudad de Cuenca-Ecuador.

Población y métodos: en el estudio descriptivo participaron 102 adultos mayores (AM) pertenecientes a los clubes de la tercera edad de los centros de salud de primer nivel. Los estudiantes del ciclo comunitario de la carrera de Medicina aplicaron un formulario validado previamente con preguntas de condiciones sociodemográficas, parámetros dietéticos y hábitos alimenticios, previa capacitación y firma del consentimiento informado.

Resultados: la edad promedio fue de $76 \pm 7.20$ años, de entre 65 a 95 años, la mayoría eran mujeres, casadas, jubiladas, con educación primaria, desocupadas y de estrato socioeconómico bajo. Casi todos ingieren desayuno, almuerzo y merienda, con una consistencia normal. Uno de cada cuatro AM ingiere suplementos vitamínicos, minerales y hierbas. Eligen los alimentos por sabor y menor costo. Ocho de cada diez no pueden preparar sus alimentos. Seis de cada diez se alimentan en forma deficiente, sin necesidad que les agrade o les desagrade lo que comen.

Conclusiones: los parámetros y hábitos alimenticios de los AM están relacionados con el envejecimiento y varios factores determinantes. Su conocimiento servirá para plantear propuestas sobre alimentación saludable y prevenir enfermedades relacionadas.

Palabras clave: anciano, nutrición, alimentación y dieta, conducta alimentaria, epidemiologia descriptiva, factores de riesgo. 


\section{ABSTRACT}

Objective: to describe the dietary parameters and eating habits of the elderly who were treated in health centers of the first level, in the city of Cuenca-Ecuador.

Population and methods: the descriptive study involved 102 elderly adults belonging to the senior clubs of the first level health centers. The students of the Community Cycle of the medical career applied a validated form, with questions of sociodemographic conditions, dietary parameters and eating habits, with prior training and signing of the informed consent.

Results: the average age was $76 \pm 7.20$ years, between 65 and 95 years, the majority was, married and retired women with primary education, they were unemployed and with low socioeconomic status. Almost everyone eats breakfast, lunch and dinner, with a normal consistency. One in four AM ingests vitamin, mineral and herbal supplements. They choose foods by taste and lower cost. Eight in ten people cannot prepare their food. Six in ten people feed poorly, it does not matter if they like or dislike what they eat.

Conclusions: the parameters and eating habits of the $A M$ are related to aging and several determining factors. The knowledge will serve to propose healthy eating and prevent related diseases.

Key words: aged, diet, food and nutrition, feeding behavior, epidemiology descriptive, risk factors.

\section{INTRODUCCIÓN}

A nivel mundial, entre 2015 y 2030 la población de 60 años y más se elevará de 900 millones a más de 1.400 millones de personas. El porcentaje de población de 60 años y más pasará del $12.3 \%$ en 2015 al $16.4 \%$ en 2030. En América Latina y El Caribe en 2037 la proporción de personas mayores sobrepasará a la proporción de menores de 15 años. En valores absolutos, la población de 60 años y más, compuesta en la actualidad por unos 76 millones de personas, tendrá un período de amplio incremento que la llevará a alcanzar las cifras de 147 millones de personas en 2037 y 264 millones en 2075 [1]. En nuestro país, según datos del último censo, de los 14'483.499 ecuatorianos, el $6.5 \%$ son AM 813.624 ; de los cuales $53 \%$ son mu- jeres y $47 \%$ hombres, y se estima que para el 2050 representen el $18 \%$ [2].

De acuerdo con la Organización Panamericana de la Salud (OPS), un envejecimiento saludable se relaciona con la prevención de las enfermedades y de la discapacidad, una nutrición adecuada, la actividad física constante y el mantenimiento de las funciones cognoscitivas, así como la participación en actividades sociales y productivas [3].

En el presente estudio abordamos el problema acerca de los parámetros dietéticos y hábitos alimenticios de los adultos mayores, para conocer, en primer lugar, los detalles sobre alimentación, consistencia de la comida, suplementos, elección de los alimentos, si pueden comprar y preparar por sí solos, la preparación más habitual y la forma de alimentarse; en segundo lugar, si los diferentes tipos de alimentos los AM consumen de acuerdo con la cantidad recomendada; y en tercer lugar, cuanto les agrada los alimentos que ingieren. Con base en los hallazgos se podrán plantear intervenciones adecuadas a la realidad local.

Una buena nutrición es fundamental para mantener la funcionalidad y la calidad de vida en los AM; no obstante, representan el grupo con mayor riesgo de alimentación inapropiada, debido a los cambios fisiológicos propios del envejecimiento, así como la presencia de enfermedades crónicas que pueden producir cambios en los hábitos alimentarios, y a su vez pueden provocar desnutrición u obesidad [4-6].

Según la evidencia científica los requerimientos nutricionales para los AM serían las siguientes:

- Los hidratos de carbono han de constituir el 50 a $60 \%$ del aporte energético total de la dieta, con un aporte equilibrado entre los hidratos de carbono simples o azúcares (10 a 12\%) y los hidratos de carbono complejos como los almidones (85 a $90 \%$ ) [7,8]; para esto, es necesario limitar el consumo de azúcar libre a menos del $10 \%$ de la ingesta calórica total $[9,10]$.

- Las grasas deberían contribuir con el 30 a 35\% del aporte energético total para evitar un aumento de peso, lo que implica disminuir el consumo de grasas saturadas generalmente de origen animal (carne, embutidos, leche entera, mantequilla, etcétera) o de algunos vegetales (coco y palma), con este tipo de grasas no se debe so- 
brepasar el $7-10 \%$ del aporte energético total; el resto del aporte energético, hasta el $30 \%$ se efectuará a expensas de mono o poli-insaturadas, para consumir grasas mono-insaturadas de origen vegetal o animal (aceite de oliva, aceitunas, aguacate, pollo), grasas poli-insaturadas (aceite de girasol, de soja, cacahuetes, pescado azul] y eliminar gradualmente las grasas industriales de tipo trans (margarina, la manteca, galletas, bollos, papas fritas o chips y otras frituras) [11].

- Las proteínas deben constituir el 12-15\% del aporte energético total (1-1.2 $\mathrm{g} / \mathrm{kg}$ de peso), se efectuará con alimentos animales (carne, pescados, aves, huevos y leche), por su aporte de aminoácidos esenciales, junto a alimentos con proteínas vegetales (legumbres, patatas, pan, pasta, arroz, cereales y los frutos secos), guardando un equilibrio entre proteínas animales y vegetales de al menos 60/40, siendo lo óptimo una relación 1/1 [12-14].

- Las necesidades diarias de líquidos oscilan entre 30 a $35 \mathrm{ml}$ por kilogramo de peso y día, o bien 1-1.5 ml por kilocaloría ingerida y día, en unas condiciones normales; aproximadamente 2-2.5 litros diarios (un litro se ingiere con los alimentos y el resto con bebidas), los requerimientos varían según la actividad, condiciones ambientales, tipo de dieta, ingesta de alcohol, presencia de enfermedades e ingesta de medicamentos [8].

- Se recomienda una ingesta diaria de 20-35 gramos de fibra, de preferencia en forma natural con los alimentos, es útil tanto para prevenir el estreñimiento como para mejorar los niveles de glucemia, el colesterol y las grasas [8].

- Mantener el consumo de sal por debajo de 5 gramos diarios [15].

- Las necesidades de minerales y vitaminas de las personas mayores sanas son similares a las de los adultos, salvo ante determinados problemas de salud añadidos como la desnutrición, el alcoholismo o por la toma de múltiples medicamentos [16].

Las repercusiones orgánicas como consecuencia de una alimentación deficiente pasan desapercibidas en etapas iniciales y solamente es posible detectar cuando se realizan exámenes médicos y de laboratorio, por lo que en la última fase se detecta el estado carencial, mediante síntomas y signos. De ahí la importancia de promocionar una alimentación saludables y prevenir la malnutrición en los adultos mayores $[17,8]$.

\section{POBLACIÓN Y MÉTODO}

Se realizó un estudio descriptivo, que contó con la aprobación del Comité de Bioética en Investigación del Área de la Salud de la Universidad de Cuen$\mathrm{ca}$, en el que participaron 102 adultos mayores, 19 hombres y 83 mujeres, pertenecientes a clubes de la tercera edad de las comunidades que fueron conformados por los docentes y estudiantes del ciclo comunitario del internado rotativo de la carrera de Medicina en los centros de salud de Cumbe (19), Ochoa León (28), Uncovía (19) y Victoria del Portete (36), pertenecientes al Ministerio de Salud Pública (MSP) y Seguro Social Campesino (SSC). Los participantes fueron seleccionados de acuerdo con los siguientes criterios de inclusión: 65 años y más de edad, de ambos sexos, sin deterioro cognitivo (evaluado mediante test de Pfeiffer), quienes expresaron su voluntad de participar y firmaron un consentimiento informado; no fue excluido ningún adulto mayor. La variables incluidas en el estudio fueron: características demográficas (edad, sexo, estado civil, con quien vive), socioeconómicas (auto-identificación, nivel de instrucción, ocupación, estrato socioeconómico), parámetros dietéticos (alimentación, consistencia de la comida, suplementos, elección de los alimentos, si puede comprar y preparar los alimentos, preparación habitual y forma de alimentarse), alimentación habitual según tipo de alimentos y cuánto le agrada los diferentes tipos de alimentos (lácteos, cereales y patatas, legumbres, verduras y hortalizas, frutas, carnes y aves, pescados, huevos, grasas y bebidas). La recolección de datos, fue realizada por los estudiantes del ciclo comunitario de la carrera de Medicina durante el período lectivo septiembre de 2018 a febrero de 2019, previo un proceso de capacitación, mediante un formulario con validación de constructo. Para garantizar la calidad de la información los docentes y tutores supervisaron el llenado de los formularios y constataron la veracidad de la información. El análisis de las variables cuantitativas se realizó mediante frecuencias, promedio, valor mínimo y máximo y desviación estándar, las variables cualitativas se analizaron con frecuencias y porcentajes y también los resultados se expusieron en gráficos. Para el análisis estadístico se utilizó el programa Excel 2010. 


\section{RESULTADOS}

\section{Tabla No 1}

Características demográficas y socioeconómicas de los adultos mayores

\begin{tabular}{|c|c|c|}
\hline Edad & $\mathbf{n}$ & $\%$ \\
\hline 65 a 79 & 70 & 68.63 \\
\hline 80 a 84 & 20 & 19.61 \\
\hline 85 y más & 12 & 11.76 \\
\hline \multicolumn{3}{|l|}{ Sexo } \\
\hline Masculino & 19 & 18.63 \\
\hline Femenino & 83 & 81.37 \\
\hline \multicolumn{3}{|l|}{ Estado Civil } \\
\hline Soltero & 15 & 14.71 \\
\hline Casado & 52 & 50.98 \\
\hline Viudo & 29 & 28.43 \\
\hline Divorciado & 6 & 5.88 \\
\hline \multicolumn{3}{|l|}{ Con quien vive } \\
\hline Solo & 34 & 33.33 \\
\hline Con esposo/a & 41 & 40.20 \\
\hline Con hijos & 30 & 29.41 \\
\hline Con hermanos & 2 & 1.96 \\
\hline Con otros familiares & 12 & 11.76 \\
\hline \multicolumn{3}{|l|}{ Jubilado } \\
\hline $\mathrm{Si}$ & 72 & 70.59 \\
\hline No & 30 & 29.41 \\
\hline \multicolumn{3}{|l|}{ Nivel de instrucción } \\
\hline Ninguno & 33 & 32.35 \\
\hline Centro de alfabetización & 9 & 8.82 \\
\hline Primaria & 56 & 54.90 \\
\hline Secundaria & 4 & 3.92 \\
\hline \multicolumn{3}{|l|}{ Estrato socioeconómico } \\
\hline Alto & 0 & 0.00 \\
\hline Medio alto & 1 & 0.98 \\
\hline Medio bajo & 4 & 3.92 \\
\hline Bajo & 62 & 60.78 \\
\hline Marginal & 35 & 34.31 \\
\hline
\end{tabular}

Fuente: base de datos Elaboración: los autores

Cerca de la mitad de AM entrevistados pertenecen al grupo etario de 65 a 74 años, con un promedio de 76 años, una edad máxima de 95 y mínima de 65 años, con un DS de 7.20, siendo la mayoría del sexo femenino. Cuatro de cada diez AM viven con su esposo/a, la tercera parte viven solos y en la misma proporción con hijos; uno de cada diez vive con otros familiares y en menor proporción con sus hermanos. La mayoría son jubiladas/os. Más de la mitad de los AM han alcanzado los estudios primarios, la tercera parte no han tenido ningún estudio formal, uno de cada diez ha estudiado en un centro 
de alfabetización y muy pocos han conseguido la formación secundaria. En lo referente a la estratificación socioeconómica, la mayoría de los entrevistados se ubican en el estrato bajo, la tercera parte en el marginal, muy pocos se ubican en el estrato medio bajo y solamente una persona alcanza el estrato medio alto (ver Tabla № 1).

Tabla No2

Parámetros dietéticos

\begin{tabular}{|c|c|c|}
\hline Alimentación & $\mathbf{n}$ & $\%$ \\
\hline Desayuno & 102 & 100.00 \\
\hline A media mañana & 41 & 40.20 \\
\hline Almuerzo & 101 & 99.02 \\
\hline Por la tarde & 47 & 46.08 \\
\hline Merienda & 88 & 86.27 \\
\hline Cena & 0 & 0.00 \\
\hline \multicolumn{3}{|l|}{ Consistencia de la comida } \\
\hline Normal & 90 & 88.24 \\
\hline Blanda & 12 & 11.76 \\
\hline Chancada & 0 & 0.00 \\
\hline Líquida & 0 & 0.00 \\
\hline \multicolumn{3}{|l|}{ Suplementos } \\
\hline Vitaminas y minerales & 27 & 26.47 \\
\hline Proteínas & 0 & 0.00 \\
\hline Hierbas & 27 & 26.47 \\
\hline Ninguno & 48 & 47.06 \\
\hline \multicolumn{3}{|l|}{ Elije su alimento por } \\
\hline Sabor & 43 & 42.16 \\
\hline Precio barato & 46 & 45.10 \\
\hline Agradable a la vista & 2 & 1.96 \\
\hline Por sus nutrientes & 15 & 14.71 \\
\hline \multicolumn{3}{|c|}{ Puede comprar y preparar la comida } \\
\hline $\mathrm{Si}$ & 16 & 15.69 \\
\hline No & 86 & 84.31 \\
\hline \multicolumn{3}{|c|}{ Preparación más habitual de alimentos } \\
\hline Frito & 6 & 5.88 \\
\hline Al vapor, hervido o estofado & 93 & 91.18 \\
\hline Asados o a la plancha & 3 & 2.94 \\
\hline \multicolumn{3}{|l|}{ La forma de alimentarse } \\
\hline Solo/a sin dificultad & 100 & 98.04 \\
\hline Solo/a con dificultad & 2 & 1.96 \\
\hline Necesita ayuda para comer & 0 & 0.00 \\
\hline
\end{tabular}

Fuente: base de datos Elaboración: los autores 
En la Tabla № 2 podemos apreciar que los parámetros dietéticos que prefieren los AM, son en su mayoría las tres comidas principales: desayuno, almuerzo y merienda, algo menos de la mitad consumen alimentos por la tarde y a media mañana y ninguno acostumbra la cena. En cuanto a la consistencia de la comida, la mayoría prefieren una preparación normal, muy pocos consumen dieta blanda y nadie consume alimentos chancados o preparados en forma líquida. Con respecto a los suplementos, uno de cada cuatro AM consume vitaminas y minerales y en igual proporción las hierbas, generalmente en infusiones. La elección de los alimentos principalmente se da por el menor costo y el sabor y en menor proporción por sus nutrientes y si es agradable para la vista. En lo referente a la adquisición y preparación de la comida, ocho de cada diez AM han manifestado que no lo pueden hacer solos. La preparación más habitual de alimentos preferida para casi todos es al vapor, hervido o estofado, a muy pocos les gusta fritos, asado o a la plancha. En cuanto a la forma de ali- mentarse, casi todos comen solos sin dificultad y únicamente dos refirieron que comen solos con dificultad.

De acuerdo con los datos expresados en el Gráfico $N^{\circ} 1$, algo más de la tercera parte de AM ingieren una cantidad adecuada de los diferentes tipos de alimentos; esto significa que la mayoría de AM se alimentan deficientemente y una minoría en exceso. Entre los alimentos que la mayoría de adultos mayores consumen de manera adecuada están los cereales y patatas, legumbres y grasas; en tanto que los alimentos que mayoritariamente consumen de manera deficiente, en orden porcentual están: el pescado, carnes y aves, frutas, lácteos, bebidas, verduras y hortalizas. Los alimentos que, entre 10 al $20 \%$ de AM consumen en exceso son: legumbres, cereales y patatas y huevos; y los alimentos como las grasas, bebidas, carnes y aves, frutas, verduras y hortalizas, consumen en exceso menos del $10 \%$ de AM.

\section{Gráfico № 1}

Alimentación habitual de los adultos mayores. Cuenca, 2019

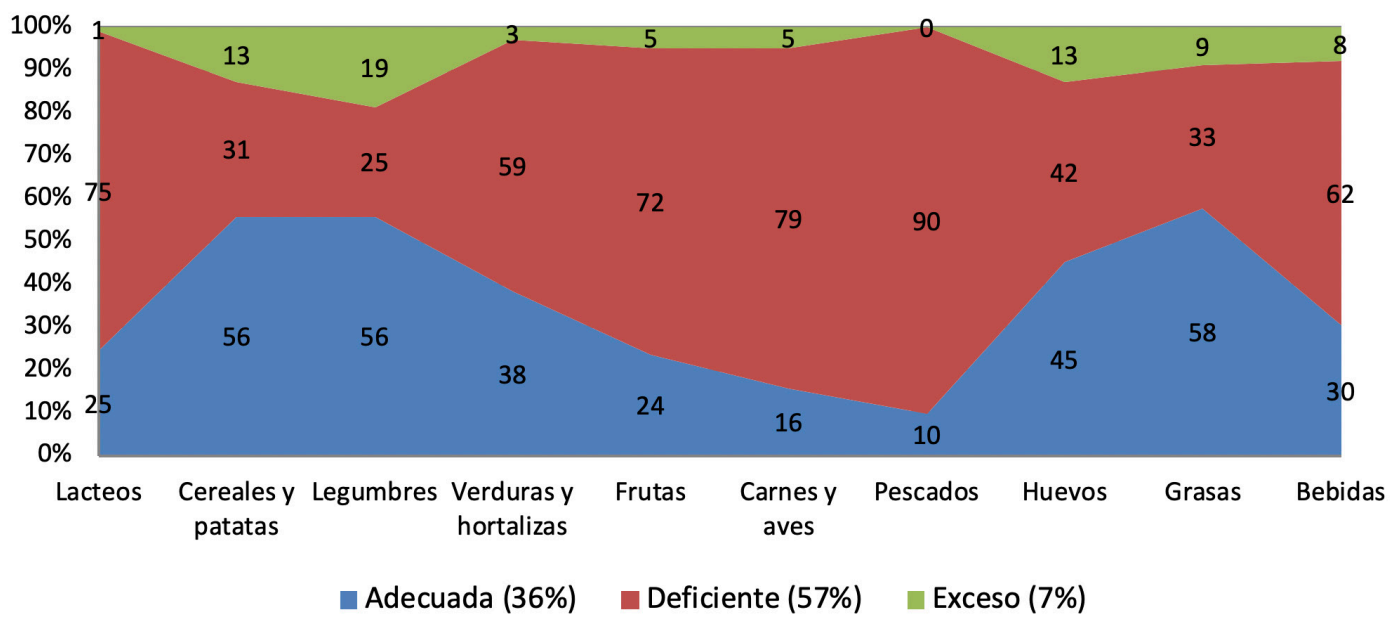

Fuente: base de datos

Elaboración: los autores 


\section{Tabla $\mathrm{N}^{\circ} 4$}

Cuanto les agrada los alimentos a los adultos mayores. Cuenca, 2019

\begin{tabular}{lcccccc}
\hline & \multicolumn{2}{c}{ Le agrada } & \multicolumn{2}{c}{ Ni le agrada ni le des- } & \multicolumn{2}{c}{ No le agrada } \\
Tipos de alimentos & $\mathbf{n}$ & $\mathbf{\%}$ & $\mathbf{n}$ & $\mathbf{\%}$ & $\mathbf{n}$ & $\mathbf{\%}$ \\
\hline Lácteos & 14 & 13.73 & 79 & 77.45 & 9 & 8.82 \\
$\begin{array}{l}\text { Cereales y } \\
\text { patatas }\end{array}$ & 15 & 14.71 & 84 & 82.35 & 3 & 2.94 \\
Legumbres & 11 & 10.78 & 87 & 85.29 & 4 & 3.92 \\
Verduras y & 14 & 13.73 & 85 & 83.33 & 3 & 2.94 \\
hortalizas & 12 & 11.76 & 87 & 85.29 & 3 & 2.94 \\
Frutas & 20 & 19.61 & 67 & 65.69 & 15 & 14.71 \\
Carnes y aves & 16 & 15.69 & 79 & 77.45 & 7 & 6.86 \\
$\begin{array}{l}\text { Pescados } \\
\text { Huevos }\end{array}$ & 14 & 13.73 & 77 & 75.49 & 11 & 10.78 \\
$\quad$ Grasas & 34 & 33.33 & 43 & 42.16 & 25 & 24.51 \\
$\quad$ Bebidas & 23 & 22.55 & 76 & 74.51 & 3 & 2.94 \\
\hline Dieta total [promedio] & $\mathbf{1 7}$ & $\mathbf{1 6 . 9 6}$ & $\mathbf{7 6}$ & $\mathbf{7 4 . 9 0}$ & $\mathbf{8}$ & $\mathbf{8 . 1 4}$ \\
\hline
\end{tabular}

Fuente: base de datos

Elaboración: los autores

Con respecto al gusto por las comidas en general, tres de cada cuatro AM manifestaron que los alimentos que ingieren ni les agrada, ni les desagrada, solamente uno de cada seis refiere que son de su gusto y que consume y el $8 \%$ se alimenta sin que le agrade lo que come. Entre los alimentos que más les agrada, en orden porcentual están: las grasas, bebidas, carnes y aves, pescados, cereales y patatas, huevos, verduras y hortalizas, lácteos, frutas y en último lugar las legumbres (Tabla $\mathrm{N}^{\circ} 4$ ).

\section{DISCUSIÓN}

Si bien algunos autores consideran, por un lado, que el uso de las encuestas dietéticas constituyen un buen método para valorar la alimentación habitual y si los parámetros dietéticos de una población están de acuerdo con los requerimientos nutricionales recomendados [18]; por otro lado, en otros estudios se cuestiona el uso del formulario, dada la complejidad de los determinantes y patrones alimentarios [19]; no obstante, consideramos que la clasificación de las personas en cuanto a su alimentación, es suficiente para saber si una población tiene o no una alimentación saludable y su posible asociación con enfermedades relacionadas con la malnutrición.
En cuanto a los parámetros dietéticos, en nuestro estudio, el $85 \%$ de AM consumen las tres comidas principales: desayuno, almuerzo y merienda, y el $43 \%$ ingieren alimentos por la tarde y a media mañana y ninguno acostumbra la cena, este porcentaje es insuficiente considerando que el déficit en el consumo de calorías y nutrientes se asocia a los procesos de fragilización. Datos similares encontramos en otros estudios; así, en Chile, el $66 \%$ realizaba tres tiempos de comida al día y un $34 \%$ consumía las cuatro [20]; en Colombia, cerca del $50 \%$ consumieron diariamente entre uno y tres veces [21].

En nuestro estudio, el menor costo de la comida como pauta de elección da cuenta de la crisis económica que influye en la deficiente alimentación de Ios AM; este criterio concuerda con lo reportado en varios estudios [20-22].

Por otro lado, en el presente estudio se detectó que el $75 \%$ de los AM consumen los alimentos sin que les agrade o les desagrade; al respecto la evidencia científica indica que la ausencia de piezas dentales, la disminución del sentido del gusto y del olfato, que reducen la sensación placentera asociada a la ingesta, la alteración de la motilidad 
gastrointestinal, que da lugar a disfagia, distensión gástrica y saciedad; el predominio de hormonas anorexígenas (colecistoquinina, polipéptido Y, leptina...) y la disminución de la secreción de insulina, con aumento de la resistencia periférica e intolerancia secundaria a la glucosa, influyen en este sentido y en la nutrición en general [23].

En este estudio, entre los alimentos que los AM consumen de manera deficiente, están: el pescado, carnes y aves, frutas, lácteos, bebidas, verduras y hortalizas; al respecto se hallaron similitudes con diversos estudios realizados en España [24,25], México [26], Perú [27] ; en tanto que en Colombia se encontró mayor consumo de frutas $(66.3 \%)$ y verduras $(57.8 \%)$ [21]. En otras investigaciones realizadas en España se reportan que los AM de condición socioeconómica media y alta, ingieren en forma adecuada frutas y verduras [22], pescado [28], carnes y grasas [25].

Hay que tener en cuenta que el pescado es considerado el más sano de las carnes blancas, bajo en grasas y calorías, fuente importante de vitaminas del complejo B y D; además, su consumo disminuye el nivel de triglicéridos y colesterol sanguíneo, reduciendo así el riesgo de enfermedades cardiovasculares, aumentando el desarrollo cognitivo en todas las etapas de la vida [29]. La baja ingesta de calcio genera pérdida mineral ósea y un mayor riesgo de fracturas, ya que el calcio y la vitamina $D$ protegen contra la osteoporosis y reducen la tasa de pérdida ósea en los últimos años de vida [23]. El consumo deficiente de frutas, verduras y hortalizas, nos señala un bajo aporte de proteína vegetal y carbohidratos, fibra, vitaminas del complejo B, especialmente B3 y ácido fólico y minerales como hierro, calcio y fósforo [20].

No obstante; en los resultados de esta investigación que se refiere al grupo de estudio con ciertas características particulares, se esperaría, que los AM con similares condiciones socioeconómicas, en especial educacionales, presentaran patrones similares respecto a las variables estudiadas. En este contexto, la mayor posibilidad de las personas mayores por mantener una alimentación saludable, estaría principalmente asociada a la educación; es por esto, que recomendamos intervenir con programas educativos en los grupos menos favorecidos educacionalmente, en lo que se refiere a los parámetros dietéticos con una activa participación comunitaria, como protectores de la salud.

\section{CONCLUSIONES}

Los adultos mayores pertenecientes a los clubes de la tercera edad, vinculados a los centros de salud de primer nivel, se alimentan en forma deficiente.

Los parámetros y hábitos alimenticios de los AM están relacionados con el envejecimiento y varios factores determinantes. Su conocimiento servirá para plantear propuestas sobre alimentación saludable y prevenir enfermedades relacionadas con la malnutrición.

\section{INFORMACIÓN DE LOS AUTORES}

- Ortiz Segarra José Ignacio, PhD. Universidad de Cuenca. Facultad de Ciencias Médicas. Internado Rotativo. Cuenca - Azuay - Ecuador.

e-mail: jose.ortiz@ucuenca.edu.ec ORCID: https://orcid.org/0000-0001-9650-1341.

- Freire Argudo Ulises Vicente. Magister en Salud Pública. Universidad de Cuenca, Facultad de Ciencias Médicas, Internado Rotativo. Cuenca Azuay - Ecuador.

e-mail: ulises.freire@ucuenca.edu.ec

ORCID: https://orcid.org/0000-0002-0942-1755

- Tenemaza Alvarracín David Fernando. Especialista en Medicina Familiar. Universidad de Cuenca. Facultad de Ciencias Médicas. Internado Rotativo. Cuenca - Azuay - Ecuador.

e-mail: david.tenemaza@ucuenca.edu.ec

ORCID: https://orcid.org/0000-0002-8330-2201

- Cobos Torres Ángel Ignacio. Magister en Salud Pública. Universidad de Cuenca. Facultad de Ciencias Médicas. Internado Rotativo. Cuenca Azuay - Ecuador.

e-mail: ignacio.cobos@ucuenca.edu.ec ORCID: https://orcid.org/0000-0003-3580-0940

- Ortiz Mejía Pedro Fernando. Médico. Libre ejercicio. Cuenca - Azuay - Ecuador.

e-mail: pedrom1820@hotmail.com

ORCID: https://orcid.org/0000-0001-8067-1389

\section{CONTRIBUCIÓN DE LOS AUTORES}

Los autores declaran haber contribuido de manera similar en la concepción, diseño, análisis e interpretación de los datos, redacción del manuscrito y aprobación de la versión final, así como estar en capacidad de responder de todos los aspectos del mismo. 


\section{CONFLICTO DE INTERESES}

Los autores declaran no tener ningún conflicto de intereses.

\section{FUENTES DE FINANCIAMIENTO}

Facultad de Ciencias Médicas de la Universidad de Cuenca por las horas de trabajo asignadas a la investigación.

\section{REFERENCIAS BIBLIOGRÁFICAS}

1. United Nations. Envejecimiento, Personas Mayores y Agenda 2030 para el Desarrollo Sostenible: Perspectiva Regional y de Derechos Humanos. Huenchuan S, editor. UN; 2019 (ECLAC Books). Disponible en: https:// www.un-ilibrary.org/economic-and-social-development/envejecimiento-personas-mayores-y-agenda-2030-para-el-desarrollo-sostenible_19532890-es

2. Instituto Nacional de Estadísticas y Censos (INEC). Salud, Bienestar y envejecimiento 2009. Disponible en: www.ecuadorencifras.gob.ec/wp-content/plugins/download.../ download.php?id...0

3. Organización Mundial de la Salud. Informe mundial sobre el envejecimiento y la salud. OMS; 2015. Disponible en: https://apps.who.int/iris/bitstream/handle/10665/186466/9789240694873_spa. pdf;jsessionid=C52EDD37BB98BE0BF3AA892797C922BB? sequence $=1$

4. Davies N. Promoting healthy ageing: the importance of lifestyle. Nurs Stand. 2011;25(19):43-9.

5. Alvarado-García A, Lamprea-Reyes L, Murcia-Tabares K. La nutrición en el adulto mayor: una oportunidad para el cuidado de enfermería. Enferm Univ. 2017;14(3):199-206.

6. Nykänen I, Rissanen TH, Sulkava R, Hartikainen $\mathrm{S}$. Effects of individual dietary counseling as part of a Comprehensive Geriatric Assessment (CGA) on nutritional status: A population-based intervention study. J Nutr Health Aging. 2014;18(1):54-8.

7. Araki $\mathrm{A}$, Ito $\mathrm{H}$. Diabetes mellitus and geriatric syndromes. Geriatr Gerontol Int. junio de 2009;9(2):105-14.
8. Gil Gregorio P. Nutrición en el anciano: guía de buena práctica clínica en geriatría. Madrid: Sociedad Española de Geriatría y Gerontología ICM Comunicación; 2014.

9. Tremayne P, Harrison P. Gastrointestinal care for older people. Nurs Stand. 2016;30(45):5363.

10. Sampson G. Weight loss and malnutrition in the elderly - The shared role of GPs and APDs. Aust Fam Physician. 2009;38(7):50710.

11. Li C-L, Chang H-Y, Wang H-H, Bai Y-B. Diabetes, functional ability, and self-rated health independently predict hospital admission within one year among older adults: A population based cohort study. Arch Gerontol Geriatr. 2011;52(2):147-52.

12. Morley JE. Decreased Food Intake With Aging. J Gerontol A Biol Sci Med Sci. 2001;56(Supplement 2):81-8.

13. Starke J, Schneider H, Alteheld B, Stehle P, Meier R. Short-term individual nutritional care as part of routine clinical setting improves outcome and quality of life in malnourished medical patients. Clin Nutr. 2011;30(2):194201. doi: 10.1016/j.clnu.2010.07.021.

14. Baldwin C, Weekes CE. Dietary advice with or without oral nutritional supplements for disease-related malnutrition in adults. Cochrane Cystic Fibrosis and Genetic Disorders Group, editor. Cochrane Database Syst Rev.2011 (9):CD002008. doi: 10.1002/14651858. CD002008.pub4. Disponible en: http://doi. wiley.com/10.1002/14651858.CD002008. pub4

15. Johnson MA. Strategies to improve diet in older adults. Proc Nutr Soc. 2013;72(1):16672. doi: $10.1017 /$ S0029665112002819..

16. Schwartz AV. Association of BMD and FRAX Score with risk of fracture in older adults with type 2 Diabetes. JAMA. 2011;305[21]:2184.

17. Gázquez Linares JJ. Salud y cuidados en el envejecimiento. Volumen III Volumen III. La Cañada de San Urbano, Almería: ASUNIVEP; 2015.

18. Banegas JR, Serra-Majem L, Rabat JM, Artalejo FR, Sabaté J, Vázcluez C, et al. Di- 
rectrices para la elaboración de estudios poblacionales de alimentación y nutrición. Rev Sanid E Hig Pública. 1994;68(2):247/260.

19. Cuñat A. et. al. Hábitos alimentarios en personas mayores de 65 años del area Sanitaria de Guadalajara, sin deterioro cognitivo y residentes en la comunidad. Rev Esp Salud Pública 2000;74(3)287-298. Disponible en http:// www.redalyc.org/articulo.oa? $\mathrm{id}=17074307$

20. Barrón $\mathrm{V}$, Rodríguez A, Chavarría P. Hábitos alimentarios, estado nutricional y estilos de vida en adultos mayores activos de la ciudad de Chillán, Chile. Rev Chil Nutr. 2017;44(1):88.

21. Gil Toro D, Giraldo NA, Estrada Restrepo A. Ingesta de alimentos y su relación con factores socioeconómicos en un grupo de adultos mayores. Rev Salud Pública. 2017;19(3):30410.

22. Hernández, Ana, Goñi I. Calidad de la dieta de la población española mayor de 80 años no institucionalizada. Nutr Hosp. 2015;31[6]:2571-7.

23. Sociedad Española de Geriatría y Gerontología. Manual del Residente en Geriatria Madrid: Ene Life Publicidad S.A. y Editores; 2011. Disponible en: https://www.academia. edu/15854898/Manual_del_Residente_en_ Geriatria_booksmedicos

24. Bach-Faig A, Berry EM, Lairon D, Reguant $J$, Trichopoulou A, Dernini S, et al. Mediterranean diet pyramid today. Science and cultural updates. Public Health Nutr. 2011;14(12a):2274-84.

25. León-Muñoz LM, Guallar-Castillón P, Graciani A, López-García E, Mesas AE, Aguilera $M T$, et al. Adherence to the Mediterranean Diet Pattern Has Declined in Spanish Adults. J Nutr. 2012;142(10):1843-50.

26. Ávila-Funes JA, Garant M-P, Aguilar-Navarro $S$. Relación entre los factores que determinan los síntomas depresivos y los hábitos alimentarios en adultos mayores de México. Rev Panam Salud Pública.2006;19(5)321-330. Disponible en: http:// www.scielosp.org/scielo.php?script=sci_arttext\&pid=S1020-49892006000500005\&I$\mathrm{ng}=\mathrm{es} \& \mathrm{nrm}=\mathrm{iso} \& \operatorname{lng}=\mathrm{es}$
27. Sánchez Ruiz F, De la Cruz Mendoza F, Cereceda Bujaico M, Espinoza Bernardo S. Asociación de hábitos alimentarios y estado nutricional con el nivel socioeconómico en adultos mayores que asisten a un Programa Municipal. An Fac Med. 2014;75(2):107-11.

28. González CA, Argilaga S, Agudo A, Amiano $\mathrm{P}$, Barricarte A, Beguiristain JM, et al. Diferencias sociodemográficas en la adhesión al patrón de dieta mediterránea en poblaciones de España. Gac Sanit. 2002;16(3):214-21.

29. Serrano Rios M. Guía de alimentación para personas mayores. Madrid: Ergon; 2010. Disponible en http://www.institutodanone.es/ assets/guia_de_alimentacion_para_personas_mayores.pdf. 